\title{
Beat-Class Set Classes and the Power Group Enumeration Theorem
}

\author{
Robert W. Peck \\ Louisiana State University \\ rpeck@1su.edu \\ Orcid: 0000-0002-6226-1631
}

DOI: $10.46926 /$ musmat.2021v5n1.01-13

\begin{abstract}
The theory of beat-class sets originates in the work of Milton Babbitt, who demonstrates a correspondence between modular pitch-class spaces and metric spaces in the framework of total serialism. Later authors, particularly Richard Cohn, John Roeder, and Robert Morris, apply similar concepts to a variety of analytical situations, drawing on concepts and procedures from pitch-class set theory. In light of the correspondence between these theories, the universe of beat-class sets for a given modulus may be partitioned into equivalence classes similar to pitch-class set classes. This study investigates processes of enumerating these equivalence classes.

We consider extensions to the theory of beat-class sets by including rhythms with more than one voice. Specifically, we examine equivalence classes of multiple-voiced beat-class sets using the Power Group Enumeration Theorem (PGET) of Frank Harary and Edgar M. Palmer. The PGET allows us to determine the numbers of equivalence classes of beat-class sets as determined by various groups of transformations: metric shift, retrogradation, and voice permutation, among others. Our results have implications for further applications in pitch-class set theory, serial theory, and transformational theory.
\end{abstract}

Keywords: Beat-class set. Equivalence class. Combinatorics. Enumeration. Power group.

\section{INTRODUCTION}

onsider rhythms (a)-(c) in Figure 1. Each rhythm displays six eighth notes, distributed among three voices. Rhythms (a) and (b) relate by a metric shift of three eighth notes. They are, accordingly, members of an orbit of the cyclic group of metric shifts, acting on the set of rhythms in the meter ${ }_{8}^{6}$. In contrast, rhythm (c) does not relate by metric shift to the previous two. Consequently, it is a member of a different orbit of this group; hence, it is not a member of the same equivalence class.

Such equivalence classes are similar to the $T_{n}$ set classes of pitch-class set theory. They are orbits of cyclic groups: the cyclic transposition group of order 12, in the case of the traditional, pitched $T_{n}$ classes, and the cyclic metric-shift group of order 6 , in the case of these rhythms. As with $T_{n}$ classes, these rhythmic equivalence classes vary in size, depending on the degree of symmetry of their members. The first two rhythms here display no rotational symmetry. As a result, the equivalence class to which they belong contains six such rhythms. The third rhythm, 
a)

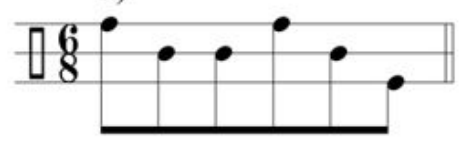

c)

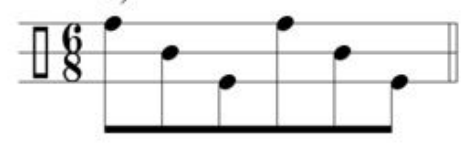

b)

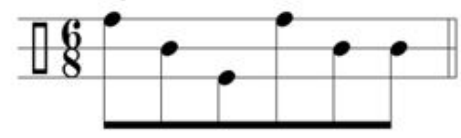

d)

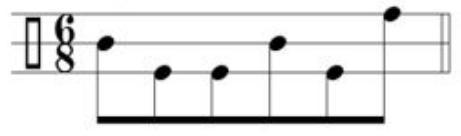

Figure 1: Four three-voiced rhythms in ${ }_{8}^{6}$.

on the other hand, is symmetrical under a metric shift of three eighth notes. As a result, its equivalence class contains only three rhythms.

Next, compare rhythm (d) in Figure 1 to (a) and (b). Rhythm (d) does not obtain from (a) by an operation on metric positions, but these rhythms do relate by voice permutation. They are members of a different equivalence class, an orbit of the group of voice permutations. Likewise, rhythms (b) and (d) are members of an equivalence class: an orbit of a group that acts both on metric positions and voices. Such a group is an example of a power group, which we investigate in greater depth below.

This study considers these types of equivalence classes. In particular, we are concerned with the enumeration of equivalence classes of rhythmic sets that relate by metric shifts and voice permutations. This task is a music-theoretical application of concepts and procedures from the mathematical field of combinatorics. Some of our work will incorporate classical combinatorial techniques, such as Burnside's Lemma and Pólya's Enumeration Theorem. Other methods derive from more recent results, including de Bruijn's Theorem and the Power Group Enumeration Theorem.

Throughout this study, we consider examples of rhythms in ${ }_{8}^{6}$ under the action of groups of metric shifts and voice permutations. Several of these examples incorporate three voices, as in Figure 1 . The theory presented here is not limited to these particular parameters. It is sufficiently general to apply to rhythms in any meter with any number of voices under the action of any relevant group. Similarly, for simplicity, the examples included here contain only beat-level onsets. The theory may apply as well to rhythms that include subdivision-level onsets.

\section{i. Beat-Class Sets}

We situate our findings in the context of beat-class set theory. The theory of beat-class sets originates in the work of Milton Babbitt [1], who refers to sets of time points in terms of a correspondence between modular pitch-class spaces and metric spaces in the framework of total serialism. Benjamin Boretz [2], John Rahn [3], and David Lewin [4] investigate this connection further. Later authors, including Richard Cohn [5], Robert Morris [6], and John Roeder [7], apply similar concepts to a variety of analytical situations, such as in the phase music of Steve Reich.

A beat-class set is a rhythmic analogue of a pitch-class set, where the modular pitch-class space of the latter is exchanged with a modular space of metric positions in the former. For instance, an eighth-note rhythm in ${ }_{8}^{6}$ has six potential beat-level onsets, which we label in the integers modulo 6 (where the downbeat is equal to 0 ). The rhythm in Figure 2 features onsets at metric positions 0 , 2 , and 3 , thereby constituting the beat-class set $\{0,2,3\}$. As with pitch-class sets, we may perform 


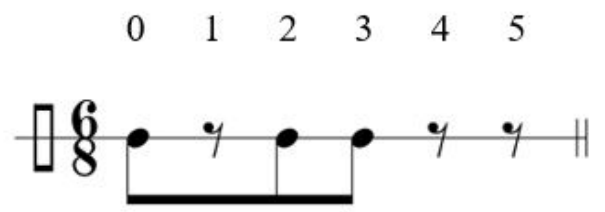

Figure 2: Beat-class set $\{0,2,3\}$.

various operations to beat-class sets. Unit metric shift generates a cyclic group that acts on the set of metric positions, similar to the group of transposition operators that acts on pitch-classes. Retrogradation agrees with the pitch-class operation of inversion. Adjoining this operation to the group of metric shifts yields a dihedral group of operators, corresponding to the pitch-class transposition-and-inversion group. The respective actions of these groups on the set of beat-class sets within a particular meter partition that set into equivalence classes that parallel the $T_{n}$ and $T_{n} / T_{n} I$ set classes of pitch-class set theory.

In this study, we consider not only beat-class sets with a single voice, like the set $\{0,2,3\}$ above, but also multiple-voice sets, such as the three-voice examples we considered initially. Operations on metric positions-such as metric shift and/or retrogradation-partition the set of multiple-voice beat-class sets into equivalence classes. However, these operations do not situate beat-class sets that are related merely by a permutation of their voices into the same equivalence class. Nevertheless, such sets are equivalent in a rhythmically generic sense. For instance, both the rhythms in Figure 1 (a) and (d) above may be described as follows: an initial voice presents an onset at metric position 0 ; two onsets follow in another voice at positions 1 and 2; the initial voice presents another single onset at position 3; the second voice has one onset at position 4 ; and a third voice concludes the rhythm with an onset at position 5. By virtue of this generic rhythmic identity, we adjoin voice permutations to the previous groups to form a new category of groups, the orbits of which constitute equivalence classes of multiple-voice beat-class sets.

In addition to the multiple-voiced rhythms in Figure 1, which contain an onset on every beat of the measure, we also consider multiple-voiced rhythms with some number of beats that contain no onsets (e.g., beats with rests or which simply contain no onset). One solution to this problem would be merely to assign the beats with no onsets to a particular voice. However, if the number of onsets is to remain constant, then we should not allow the voice with no onsets to permute with the voices with onsets. As we will see, the Power Group Enumeration Theorem offers a method for achieving this result.

\section{ii. Enumeration Applications in Music Theory}

Considering that pitch-class set theory as a discipline did not emerge until the 1960s and early 1970s, the enumeration of what are essentially pitch-class set classes has a surprisingly long tradition, beginning in the late 19th and early 20th centuries. Jonathan Bernard [8] and Catherine Nolan $[9,10]$ discuss the history of early efforts in this endeavor, citing the work of Heinrich Vincent, Anatole Loquin, and Ernst Bacon. Julian Hook [11] presents a detailed tutorial of classical combinatorial enumeration techniques, including Burnside's Lemma and Pólya's Enumeration Theorem, as applied to a host of music-theoretical topics, including the counting of $T_{n}, T_{n} / T_{n} I$, and $T_{n} / T_{n} I / T_{n} M$ set classes in modular pitch-class spaces of various sizes, row classes of twelve-tone series, equivalence classes of beat-class sets, and the like.

Other music-theoretical research that incorporates enumeration applies these techniques to 
various problems. Joel Haack [12] uses combinatorial methods to determine the number of beatclass sets that have the same properties as - and that could substitute for-the rhythm that serves as the basis for Steve Reich's 1972 composition Clapping Music. Ronald C. Read [13] considers the size of the set of all possible performances of Stockhausen's Klavierstück XI. He calculates precisely the number of paths that may be taken through 19 fragments that constitute the score to that piece, approximately equal to $1.7 \times 10^{41}$. Harald Fripertinger [14, 15] discusses the enumeration of a variety of musical objects, such as intervals and chords, patterns of rhythms and motives, as well as tone rows and patterns of tropes. Fripertinger and Peter Lackner [16] examine in greater depth the enumeration of the latter two topics in an article that constitutes an entire special issue of Journal of Mathematics and Music. The enumeration of tropes is of particular relevance to this study, as it involves the action of a power group: a group that acts on hexachordal partitions of 12-tone rows by transposing or inverting the pitch-classes in the hexachords and/or by permuting the hexachords themselves.

\section{Enumeration of Equivalence Classes of Beat-Class Sets}

\section{i. Single-Voice Sets}

The enumeration of single-voice beat-class sets, such as the example of the beat-class set $\{0,2,3\}$ above, is equivalent to counting the number of subsets of beats classes in a single measure with $n$ beats. For a given subset, two possibilities exist for any beat class in the measure: the subset may include an onset at that beat or it may not. Hence, there is a total of $2^{n}$ possible subsets. The number of equivalence classes to which these subsets belong, however, is not merely $2^{n}$ divided by the order of the group that acts on the beat classes, as certain sets may possess symmetry.

Burnside's Lemma gives the number of orbits as an average. Each member $\alpha$ of the group $A$ fixes some number of elements of the set $X$ on which the group acts, the set $X^{\alpha}$. The number of orbits for $A$, then, is the average of these numbers of fixed elements as $\alpha$ varies within $A$ :

$$
|A / G|=\frac{1}{|A|} \sum_{\alpha \in A}\left|X^{\alpha}\right|
$$

In the case of ${ }_{8}^{6}$, we observe that there exist $2^{6}=64$ possible beat class sets (including the empty set and the set that includes onsets in all six metric positions). As we noted above, some of these sets possess symmetry. To calculate the number of orbits into which the 64 sets partition under the action of the group of metric shifts, we need to know how many of these sets are fixed by translation by one metric position, two metric positions, etc., through six metric positions. Then, the number of equivalence classes is the average of these tallies.

To ascertain the numbers of sets that are fixed by any particular member of a group, it is useful to examine the disjoint cycle decomposition of each element of the group. Let $A$ be a permutation group with an action on a set $X$ of size $d$. Each permutation in $A$ may be written as a product of disjoint cycles. For every $k$ from 1 to $d$, let $j_{k}$ be a function that counts the number of cycles of length $k$ among the disjoint cycles in the decomposition of the permutation. For example, our group of metric shifts in ${ }_{8}^{6}$ acts on the set of 6 metric positions, labeled 0 (downbeat) through 5. Let $T_{n}$ be a metric shift of $n$ eighth notes. Then, the members of $A$ have the disjoint cycle 
Table 1: Values of $j_{k}\left(T_{n}\right)$.

\begin{tabular}{ccccccc}
\hline$T_{n}$ & $j_{1}$ & $j_{2}$ & $j_{3}$ & $j_{4}$ & $j_{5}$ & $j_{6}$ \\
\hline$T_{0}$ & $j_{1}\left(T_{0}\right)=\mathbf{6}$ & $j_{2}\left(T_{0}\right)=0$ & $j_{3}\left(T_{0}\right)=0$ & $j_{4}\left(T_{0}\right)=0$ & $j_{5}\left(T_{0}\right)=0$ & $j_{6}\left(T_{0}\right)=0$ \\
$T_{1}$ & $j_{1}\left(T_{1}\right)=0$ & $j_{2}\left(T_{1}\right)=0$ & $j_{3}\left(T_{1}\right)=0$ & $j_{4}\left(T_{1}\right)=0$ & $j_{5}\left(T_{1}\right)=0$ & $j_{6}\left(T_{1}\right)=\mathbf{1}$ \\
$T_{2}$ & $j_{1}\left(T_{2}\right)=0$ & $j_{2}\left(T_{2}\right)=0$ & $j_{3}\left(T_{2}\right)=\mathbf{2}$ & $j_{4}\left(T_{2}\right)=0$ & $j_{5}\left(T_{2}\right)=0$ & $j_{6}\left(T_{2}\right)=0$ \\
$T_{3}$ & $j_{1}\left(T_{3}\right)=0$ & $j_{2}\left(T_{3}\right)=\mathbf{3}$ & $j_{3}\left(T_{3}\right)=0$ & $j_{4}\left(T_{3}\right)=0$ & $j_{5}\left(T_{3}\right)=0$ & $j_{6}\left(T_{3}\right)=0$ \\
$T_{4}$ & $j_{1}\left(T_{4}\right)=0$ & $j_{2}\left(T_{4}\right)=0$ & $j_{3}\left(T_{4}\right)=\mathbf{2}$ & $j_{4}\left(T_{4}\right)=0$ & $j_{5}\left(T_{4}\right)=0$ & $j_{6}\left(T_{4}\right)=0$ \\
$T_{5}$ & $j_{1}\left(T_{5}\right)=0$ & $j_{2}\left(T_{5}\right)=0$ & $j_{3}\left(T_{5}\right)=0$ & $j_{4}\left(T_{5}\right)=0$ & $j_{5}\left(T_{5}\right)=0$ & $j_{6}\left(T_{5}\right)=\mathbf{1}$ \\
\hline
\end{tabular}

decompositions shown here:

$$
\begin{aligned}
& T_{0}:=(0)(1)(2)(3)(4)(5) \\
& T_{1}:=(0,1,2,3,4,5) \\
& T_{2}:=(0,2,4)(1,3,5) \\
& T_{3}:=(0,3)(1,4)(2,5) \\
& T_{4}:=(0,4,2)(1,5,3) \\
& T_{5}:=(0,5,4,3,2,1) .
\end{aligned}
$$

Table 1 shows the values of $j_{k}$ for each $T_{n}$ (non-zero values appear in bold).

Call $q\left(T_{n}\right)$ the sum of all values $j_{k}$ for any particular $T_{n}$. Then, we use $2^{q\left(T_{n}\right)}$ to determine the number of beat-class sets in ${ }_{8}^{6}$ that are symmetrical under the element $T_{n}$ of the group of metric shifts. For example, since $q\left(T_{0}\right)=6, T_{0}$ fixes all $2^{6}=64$ beat-class sets. Similarly, since $q\left(T_{1}\right)=1$, $T_{1}$ fixes 2 beat-class sets, and so forth. The total number of beat-class sets fixed by the elements of the group is 84 , which, divided by 6 (the size of the group), equals 14 - the number of $T_{n}$ equivalence classes (or orbits of this particular group).

It will be useful below to keep track of the disjoint cycle decomposition of the elements of a group $A$. We may do this by means of the cycle index of $A, Z(A)$, a polynomial in the variables $s_{1}, s_{2}, \ldots, s_{d}:{ }^{1}$

$$
Z(A)=\frac{1}{|A|} \sum_{\alpha \in A} \prod_{k=1}^{d} s_{k}^{j_{k}(\alpha)} .
$$

For example, consider the cycle index for the group $A$ of metric shifts in ${ }_{8}^{6}$ :

$$
Z(A)=\frac{1}{6}\left(s_{1}^{6}+s_{2}^{3}+2 s_{3}^{2}+2 s_{6}\right) .
$$

Note that there exists one element in the group, $T_{0}$, that contains six cycles of length 1 , and 1 is the coefficient of $s_{1}$ in $Z(A)$; one element, $T_{3}$, contains three cycles of length 2 , and 1 is the coefficient of $s_{2}$; two elements, $T_{2}$ and $T_{4}$, contain two cycles of length 3 , and 2 is the coefficient of $s_{3}$; finally, two elements, $T_{1}$ and $T_{5}$, contain one cycle of length 6 , and 2 is the coefficient of $s_{6}$.

Next, we wish to determine the number of equivalence classes that exist for sets of the same size. Let $A$ be a group of order $m$ that acts on a set $X$ of size $d ; \alpha \in A$. Further, let $B$ be a group that acts on a set $Y$ of size $e \geq 2$ into which we map the elements of $X ; \beta \in B$.

\footnotetext{
${ }^{1}$ The variable $s$ here does not mean anything; it is a placeholder.
} 
Definition 1. Call $B^{A}$ the power group, which has an action of the set $Y^{X}$ of all functions $f: X \rightarrow Y$. The members of $B^{A}$ are the ordered pairs $(\alpha ; \beta)$. Any function $f$ in $Y^{X}$ under $(\alpha ; \beta)$, then, consists of the mapping $((\alpha ; \beta) f)(x)=\beta f(\alpha x)$.

For now, however, we will assume that $B$ is the identity group-that is, its single element does not permute elements of $Y$. let

Let $w$ be a weight function that maps $Y$ into the set of non-negative integers, $0 \leq k \leq \infty$, and

$$
c^{k}=\left|w^{-1}(k)\right|
$$

be the number of number of configurations with weight $k$. Then, we call the series in the indeterminant $x$,

$$
c(x)=\sum_{k=0}^{\infty} c_{k} x^{k}
$$

the "configuration counting series". Because all functions in the same orbit of $B^{A}$ have the same weight, there exists a finite number of orbits of any particular weight. Therefore, let $C_{k}$ be the size of the set of orbits of weight $k$. Then, the series in the indeterminant $x$,

$$
C(x)=\sum_{k=0}^{\infty} C_{k} x^{k}
$$

is the "function counting series". This leads to the weighted version of Pólya's Enumeration Theorem. Let $Z(A, c(x))$ be an abbreviated denotation of $Z\left(A ; c(x), c\left(x^{2}\right), c\left(x^{3}\right), \ldots\right)$.

Theorem 1 (Pólya). We substitute $c\left(x^{k}\right)$ in the configuration counting series for each variable $s_{k}$ in $Z(A)$ to determine the function counting series $C(x)$. That is,

$$
C(x)=Z(A, c(x)) \text {. }
$$

Let us again consider the example of beat-class sets in ${ }_{8}^{6}$. Let $A$ be the group of metric shifts, acting on the set of six beat classes, and let $B$ be the identity group with an action on a single voice. The configuration counting series for $A$ appears here:

$$
c(x)=(1+x)+\left(1+x^{2}\right)+\left(1+x^{3}\right)+\left(1+x^{4}\right)+\left(1+x^{5}\right)+\left(1+x^{6}\right) .
$$

We recall the cycle index of $A$ from Equation 9, and we substitute $\left(1+x^{k}\right)$ for $s_{k}$ in $Z(A)$ to determine the function counting series:

$$
C(x)=\frac{1}{6}\left((1+x)^{6}+\left(1+x^{2}\right)^{3}+2\left(1+x^{3}\right)^{2}+2\left(1+x^{6}\right)\right) .
$$

Expanding the above yields the following polynomial,

$$
\begin{array}{r}
C(x)=\frac{1}{6}\left(\left(1+6 x+15 x^{2}+20 x^{3}+15 x^{4}+6 x^{5}+x^{6}\right)+\left(1+3 x^{2}+3 x^{4}+x^{6}\right)\right. \\
\left.+\left(2+4 x^{3}+2 x^{6}\right)+\left(2+2 x^{6}\right)\right)
\end{array}
$$

which we simplify as follows:

$$
C(x)=1+x+3 x^{2}+4 x^{3}+3 x^{4}+x^{5}+x^{6} .
$$


The coefficients of $x^{k}$ in the polynomial determine the number of equivalence classes for beat-class sets of size $k$. Therefore, there exists one equivalence class of sets of size 0 , one equivalence class of sets of size 1, three of sets of size 2, and so on, for a total of 14 .

\section{ii. Multiple-Voice Sets}

We may also use Pólya's Enumeration Theorem to find the numbers of equivalence classes of multiple-voiced beat-class sets. For $e$ voices, we substitute an expression with $e$ variable summands in place of the indeterminants in the cycle index of $A$. For instance, if we wish to enumerate the equivalence classes of the $3^{6}=729$ three-voice beat-class sets in ${ }_{8}^{6}$, we could replace $s_{k}$ in $Z(A)$ with $\left(1+x^{k}+y^{k}+z^{k}\right)$. Then, the coefficients of $x^{m} y^{n} z^{p}$ in $C(x, y, z)$ are the numbers of equivalence classes of sets with $m, n$, and $p$ onsets in each respective voice.

The method above for partitioning the set of $e$-voiced beat-class sets in a meter with $d$ beats does not include sets that relate to one another by a permutation of the voices in the same equivalence class. If we wish to determine the numbers of these larger equivalence classes, we utilize the Power Group Enumeration Theorem of Harary and Palmer (PGET), which generalizes Pólya's Enumeration Theorem [17]. ${ }^{2}$ The PGET comes in two forms: the constant form, which counts the total number $N$ of orbits of a power group; and the polynomial form, which enumerates the number $N(x)$ of equivalence classes of sets of the same cardinality.

We define the power group $B^{A}$ as above, where $A$ is a group of metric shifts, acting on a set $X$ of $d$ beats in a given meter. In the previous examples, however, we let $B$ be merely an identity group, acting trivially on the set of $e=1$ voices. Now, we let $B$ have a non-trivial action on the set of $e>1$ voices. Then, the PGET in its constant form is as follows:

Theorem 2 ([17], p. 163).

$$
N=\frac{1}{|B|} \sum_{\beta \in B} Z\left(A ; m_{1}(\beta), m_{2}(\beta), \ldots, m_{d}(\beta)\right)
$$

where

$$
m_{k}(\beta)=\sum_{s \mid k} s j_{s}(\beta)
$$

Let us take the case of beat-class sets in ${ }_{8}^{6}$ that include up to three voices. We are interested in determining the number of equivalence classes of these sets that are inclusive both of metric shifts and permutations of the voices. Then, $A$ is a cyclic group of order 6 , acting on the set of six beats, and $B$ is the symmetric group of degree three, the set of all permutations of three voices. We gave the cycle index of $A$ above (see Equation 9); the cycle index of $B$ appears below:

$$
Z(B)=\frac{1}{6}\left(s_{1}^{3}+3 s_{1} s_{2}+2 s_{3}\right) .
$$

There exists one element of $B$ that consists of three cycles of length 1 ; three elements of $B$ that contain one cycle of length 1 and one cycle of length 2; and two elements of $B$ that have one cycle of length 3 .

We can calculate the tuple $\left(m_{1}(\beta), m_{2}(\beta), \ldots, m_{d}(\beta)\right)$ for a member $\beta \in B$ as follows. Let $\beta$ be one of the three elements $s_{1} s_{2}$ of the group of voice permutations that has one cycle of length 1 and one cycle of length 2 . Table 2 demonstrates that the tuple $\left(m_{1}(\beta), m_{2}(\beta), \ldots, m_{d}(\beta)\right)$ for

\footnotetext{
${ }^{2}$ The PGET is itself a generalization of an earlier result, de Bruijn's Theorem, which enumerates equivalence classes of weighted functions from one set to another [18].
} 
Table 2: Calculating $\left(m_{1}(\beta), m_{2}(\beta), \ldots, m_{6}(\beta)\right)$ for $\beta=s_{1} s_{2}$.

\begin{tabular}{|c||c|c|c|r|}
\hline$m_{k}$ & $k$ & $s$ & $j_{s}(\beta)$ & value of $m_{k}(\beta)$ \\
\hline \hline$m_{1}$ & 1 & 1 & $j_{1}(\beta)=1$ & $(1 \cdot 1)=1$ \\
\hline$m_{2}$ & 2 & 1 & $j_{1}(\beta)=1$ & $(1 \cdot 1)+(2 \cdot 1)=3$ \\
& & 2 & $j_{2}(\beta)=1$ & $(1 \cdot 1)+(3 \cdot 0)=1$ \\
\hline$m_{3}$ & 3 & 1 & $j_{1}(\beta)=1$ & \\
& & 3 & $j_{3}(\beta)=0$ & \\
\hline$m_{4}$ & 4 & 1 & $j_{1}(\beta)=1$ & \\
& & 2 & $j_{2}(\beta)=1$ & \\
& & 4 & $j_{4}(\beta)=0$ & $(1 \cdot 1)+(2 \cdot 1)+(4 \cdot 0)=3$ \\
\hline$m_{5}$ & 5 & 1 & $j_{1}(\beta)=1$ & \\
& & 5 & $j_{5}(\beta)=0$ & \\
\hline$m_{6}$ & 6 & 1 & $j_{1}(\beta)=1$ & \\
& & 2 & $j_{2}(\beta)=1$ & \\
& & 3 & $j_{3}(\beta)=0$ & \\
& & 6 & $j_{6}(\beta)=0$ & $(1 \cdot 1)+(2 \cdot 1)+(3 \cdot 0)+(6 \cdot 0)=3$ \\
\hline
\end{tabular}

this particular member of $B$ is $(1,3,1,3,1,3)$. In this way, we can calculate the tuples for all the members of $B$ :

- The tuple for the one element $s_{1}^{3}$ of $B$ is $(3,3,3,3,3,3)$.

- The tuple for the three elements $s_{1} s_{2}$ of $B$ is $(1,3,1,3,1,3)$.

- The tuple for the two elements $s_{3}$ of $B$ is $(0,0,3,0,0,3)$.

Substituting each of the above for $s_{k}$ in $Z(A)$ and summing the results yields

$$
(1 \cdot 130)+(3 \cdot 6)+(2 \cdot 4)=156
$$

which, when divided by the order of $B=6$, gives 26 equivalence classes that incorporate up to three voices in ${ }_{8}^{6}$.

This number, however, also includes those equivalence classes that incorporate one and two voices. Therefore, to determine the number of equivalence classes that use exactly three voices, we may repeat the procedure, substituting the symmetric group of degree 2 for $B$, yielding 8 , and subtracting that result from 26. Hence, we find precisely 18 classes of three-voiced beat class sets in ${ }_{8}^{6}$ that are equivalent under metric shift and voice permutation. Figure 3 displays one representative rhythm from each of these equivalence classes (up to metric shift and voice permutation).

\section{iii. Beat-Class Sets of Varying Cardinalities within a Meter}

The previous process considers only multiple-voiced rhythms that feature onsets on every beat in a measure, as in our initial examples in Figure 1. If we wish to consider beat-class sets of variable cardinalities within a meter, we incorporate the polynomial form of the PGET.

Theorem 3 ([17], p. 166). The polynomial which enumerates according to weight the equivalence classes of functions in $Y^{X}$ determined by the power group $B^{A}$ is

$$
N(x)=\sum_{\beta \in B} Z\left(A ; m_{1}(\beta, x), m_{2}(\beta, x), \ldots, m_{d}(\beta, x)\right),
$$



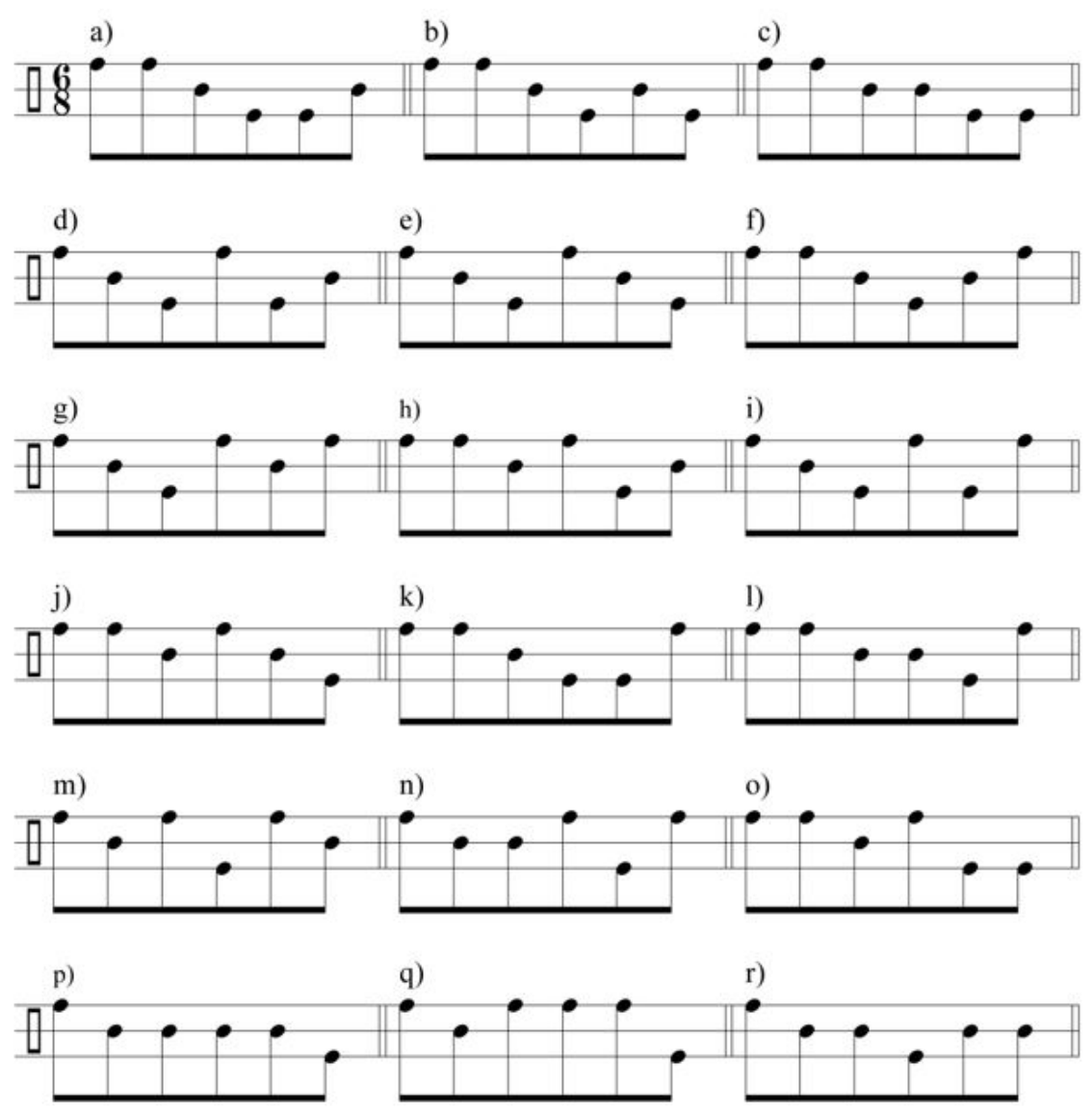

Figure 3: Representatives from each equivalence class of 3-voiced rhythms in ${ }_{8}^{6}$.

where

$$
m_{k}(\beta, x)=\sum_{t=0}^{r}\left(\sum_{s \mid k} s j_{s}\left(\beta_{t}\right)\right) x^{k t}
$$

In doing so, we assign to each voice a particular weight. We assign the beats that include no onsets to a voice with a weight of $t=0$ - essentially disabling its ability to permute with the other voices-and all other voices to a weight of $t=1$.

Let us return to our previous example of three-voiced, eighth-note rhythms in ${ }_{8}^{6}$ under the action of the power group $B^{A}$, where $A$ is the group of metric shifts and $B$ is the group of voice permutations. We now use Theorem 3 to discover how many equivalence classes exist for measures that incorporate varying numbers of beats with onsets, from 0 to 6 . In doing so, we apply the cycle index of $A$ to the tuple $\left(m_{1}(\beta, x), m_{2}(\beta, x), \ldots, m_{6}(\beta, x)\right)$ for each $\beta \in B$. Table 3 demonstrates the calculation of $\left(m_{1}(\beta, x), m_{2}(\beta, x), \ldots, m_{6}(\beta, x)\right)$ for an arbitrary member $\beta \in B$ with the form $s_{1} s_{2}$. Accordingly,

$$
\left(m_{1}(\beta, x), m_{2}(\beta, x), \ldots, m_{6}(\beta, x)\right)=\left(1+x, 1+3 x^{2}, 1+x^{3}, 1+3 x^{4}, 1+x^{5}, 1+3 x^{6}\right) .
$$

In this way, we determine the following values for each of the members of $B$. 
- The tuple for the one element $s_{1}^{3}$ of $B$ is $\left(1+3 x, 1+3 x^{2}, 1+3 x^{3}, 1+3 x^{4}, 1+3 x^{5}, 1+3 x^{6}\right)$.

- The tuple for the three elements $s_{1} s_{2}$ of $B$ is $\left(1+x, 1+3 x^{2}, 1+x^{3}, 1+3 x^{4}, 1+x^{5}, 1+3 x^{6}\right)$.

- The tuple for the two elements $s_{3}$ of $B$ is $\left(1,1,1+3 x^{3}, 1,1,1+3 x^{6}\right)$.

Substituting each of these values for $s_{k}$ in the cycle index of $A$ and summing those results for the remaining members of $B$ yields the following polynomial, where the coefficients of $x^{k}$ show the number of equivalence classes of measures that incorporate $k$ beats that contain onsets (i.e., sets of cardinality $k$ ):

$$
1+1 x+6 x^{2}+18 x^{3}+38 x^{4}+41 x^{5}+26 x^{6} .
$$

The polynomial indicates that there exists 1 equivalence class with 0 beats that contain an onset (the empty measure), 1 equivalence class with 1 onset, 6 with 2 onsets, 18 with 3 onsets, 38 with 4 onsets, 41 with 5 onsets, and 26 (the same number we found above) with 6 onsets.

The above totals include rhythms with up to three voices. If we wish to determine the number of equivalence classes in ${ }_{8}^{6}$ that include exactly three voices, we need to determine the number of equivalence classes with up to two voices (i.e., for $e=2$ ) for the sets of varying cardinality and subtract those values from the corresponding values above:

$$
\begin{array}{r}
1+1 x+6 x^{2}+18 x^{3}+38 x^{4}+41 x^{5}+26 x^{6} \\
-1+1 x+6 x^{2}+14 x^{3}+22 x^{4}+16 x^{5}+8 x^{6} \\
\hline 0+0 x+0 x^{2}+4 x^{3}+16 x^{4}+25 x^{5}+18 x^{6}
\end{array}
$$

The coefficients in the difference indicate 0 equivalence classes of beat-class sets with 0 onsets, 1 onset, or 2 onsets (as it is not possible to have exactly three voices for sets of cardinalities $0 \leq k \leq 2)$; 2 equivalence classes of sets with 3 onsets; 16 with 4 onsets; 25 with 5 onsets; and 18 with 6 onsets (again, agreeing with our previous result).

\section{iv. Results for $d \leq 6, e \leq 6$}

Table 4 presents the numbers of equivalence classes for all beat-class sets in ${ }_{8}^{6}$ of cardinalities $k$, $1 \leq k \leq 6$, that contain exactly $e$ voices, $0 \leq e \leq 6$. In each case, we determine the polynomials $N(x)$ as above for each value $k$, and subtract from the coefficients in any one polynomial the corresponding coefficients of the polynomial for the value $k-1$. We note that no beat class sets are possible with $e$ voices where $e<k$ (with the exception of $e=1$, a single voice, wherein we count the trivial case of $k=0$, the empty measure).

\section{CONCLUSIONS}

The Power Group Enumeration Theorem permits us to count objects on which exist two group actions. Such situations occur frequently in musical contexts, particularly as events-which may relate to one another by means of group actions on a space, such as transposition or inversion-also relate to one another in time, in which we define other operations. Additionally, it allows us to complete this task merely on the basis of the cycle indices of the respective group actions-information that is readily available. Whereas it requires a considerable amount of computation, it is significantly more efficient than counting these objects by hand, particularly as there exists a combinatorial explosion as the cardinalities of the sets on which the constituent groups act increases. 
Table 3: Calculating $\left(m_{1}(\beta, x), m_{2}(\beta, x), \ldots, m_{6}(\beta, x)\right)$ for $\beta=s_{1} s_{2}$

\begin{tabular}{|l||r|r|r|r|}
\hline$m_{k}$ & $t$ & $s$ & $j_{s}\left(\beta_{t}\right) x^{k t}$ & polynomial for $m_{k}(\beta, x)$ \\
\hline \hline$m_{1}$ & 0 & 1 & $1 x^{1 \cdot 0}$ & 1 \\
& 1 & 1 & $1 x^{1 \cdot 1}$ & $+1 x^{1}$ \\
\hline$m_{2}$ & 0 & 1 & $1 x^{2 \cdot 0}$ & 1 \\
& & 2 & $0 x^{2 \cdot 0}$ & +0 \\
& 1 & 1 & $1 x^{2 \cdot 1}$ & $+1 x^{2}$ \\
& & 2 & $1 x^{2 \cdot 1}$ & $+2 x^{2}$ \\
\hline$m_{3}$ & 0 & 1 & $1 x^{3 \cdot 0}$ & 1 \\
& & 3 & $0 x^{3 \cdot 0}$ & +0 \\
& 1 & 1 & $1 x^{3 \cdot 1}$ & $+1 x^{3}$ \\
& & 3 & $0 x^{3 \cdot 1}$ & $+0 x^{3}$ \\
\hline$m_{4}$ & 0 & 1 & $1 x^{4 \cdot 0}$ & 1 \\
& & 2 & $0 x^{4 \cdot 0}$ & +0 \\
& & 4 & $0 x^{4 \cdot 0}$ & +0 \\
& 1 & 1 & $1 x^{4 \cdot 1}$ & $+1 x^{4}$ \\
& & 2 & $1 x^{4 \cdot 1}$ & $+2 x^{4}$ \\
& & 4 & $0 x^{4 \cdot 1}$ & $+0 x^{4}$ \\
\hline$m_{5}$ & 0 & 1 & $1 x^{5 \cdot 0}$ & 1 \\
& & 5 & $0 x^{5 \cdot 0}$ & +0 \\
& 1 & 1 & $1 x^{5 \cdot 1}$ & $+1 x^{5}$ \\
& & 3 & $0 x^{5 \cdot 1}$ & $+0 x^{5}$ \\
\hline$m_{6}$ & 0 & 1 & $1 x^{6 \cdot 0}$ & 1 \\
& & 2 & $0 x^{6 \cdot 0}$ & +0 \\
& & 3 & $0 x^{6 \cdot 0}$ & +0 \\
& & 6 & $0 x^{6 \cdot 0}$ & +0 \\
& 1 & 1 & $1 x^{6 \cdot 1}$ & $+1 x^{6}$ \\
& & 2 & $1 x^{6 \cdot 1}$ & $+2 x^{6}$ \\
& & 3 & $0 x^{6 \cdot 1}$ & $+0 x^{6}$ \\
& & 6 & $0 x^{6 \cdot 1}$ & $+0 x^{6}$ \\
\hline
\end{tabular}

Table 4: Numbers of equivalence classes for beat-class sets in ${ }_{8}^{6}$ of cardinality $k$ with e voices

\begin{tabular}{r||rrrrrrr} 
& $k=0$ & $k=1$ & $k=2$ & $k=3$ & $k=4$ & $k=5$ & $k=6$ \\
\hline \hline$e=1$ & 1 & 1 & 3 & 4 & 3 & 1 & 1 \\
\hline$e=2$ & 0 & 0 & 3 & 10 & 19 & 15 & 7 \\
\hline$e=3$ & 0 & 0 & 0 & 4 & 16 & 25 & 18 \\
\hline$e=4$ & 0 & 0 & 0 & 0 & 3 & 10 & 13 \\
\hline$e=5$ & 0 & 0 & 0 & 0 & 0 & 1 & 3 \\
\hline$e=6$ & 0 & 0 & 0 & 0 & 0 & 0 & 1
\end{tabular}


One area for further related work is the enumeration of equivalence classes of multiple-voiced beat class sets in which we wish to keep track of how many beats are articulated in each of the respective voices. It is possible to count the sets themselves using standard combinatorial methods, but I have yet to find an efficient method for counting their equivalence classes.

\section{REFERENCES}

[1] Babbitt, M. 1962. Twelve-Tone Rhythmic Structure and the Electronic Medium. Perspectives of New Music, 1, pp. 49-79.

[2] Boretz, B. 1970. Sketch for a Musical System (Meta-Variations, Part II). Perspectives of New Music, 8, pp. 49-111.

[3] Rahn, J. 1975. On Pitch or Rhythm: Interpretations of Orderings of and in Pitch and Time. Perspectives of New Music, 13, pp. 182-203.

[4] Lewin, D. 1987. Generalized Musical Intervals and Transformations New Haven: Yale University Press.

[5] Cohn, R. 1992. Transpositional Combination of Beat-Class Sets in Steve Reich's Phase-Shifting Music. Perspectives of New Music, 30, pp. 146-77.

[6] Morris, R. 1988. Generalizing Rotational Arrays. Journal of Music Theory, 32, pp. 75-132.

[7] Roeder, J. 2003. Beat-Class Modulation in Steve Reich's Music Music Theory Spectrum, 25, pp. 275-304.

[8] Bernard, J. 1997. Chord, Collection, and Set in Twentieth-Century Theory. In Music Theory in Concept and Practice, (J. M. Baker; D. W. Beach; J. W. Bernard, ed.), pp. 11-51. Rochester: University of Rochester Press.

[9] Nolan, C. 2002. Music Theory and Mathematics. In The Cambridge History of Western Music Theory, (T. Christensen, ed.), pp. 272-304 Cambridge: Cambridge University Press.

[10] Nolan, C. 2003. Combinatorial Space in Nineteenth- and Early Twentieth-Century Music Theory. Music Theory Spectrum, 25, pp. 205-41.

[11] Hook, J. 2003. Why Are There Twenty-Nine Tetrachords? A Tutorial on Combinatorics and Enumeration in Music Theory. Music Theory Online, 13. Available at: http://https: //www.mtosmt.org/issues/mto.07.13.4/mto.07.13.4.hook.html.

[12] Haack, J. 1991. Clapping Music-A Combinatorial Problem. College Mathematics Journal, 22, pp. $224-27$.

[13] Read, R. 1997. Combinatorial Problems in the Theory of Music. Discrete Mathematics, 167-168, pp. 543-551.

[14] Fripertinger, H. 1993. Enumeration in Musical Theory. Beiträge zur elektronischen Musik, 1. Available at: https://iem.kug.ac.at/fileadmin/media/iem/altdaten/ projekte/publications/bem/bem1/bem1.pdf

[15] Fripertinger, H. 1999. Enumeration and Construction in Music Theory. In: Diderot Forum on Mathematics and Music: Computational and Mathematical Methods in Music, (H. G. Feichtinger; M. Dörfler, ed.), pp. 179-204. Vienna: Österreichische Computergesellschaft. 
[16] Fripertinger, H.; Lackner, P. 2015. Tone Rows and Tropes. Journal of Mathematics and Music, 9, pp. 111-172.

[17] Harary, F.; Palmer, E. 1966. The Power Group Enumeration Theorem. Journal of Combinatorial Theory, 1, pp. 157-173.

[18] de Bruijn, N. 1964. Pólya's Theory of Counting. In Applied Combinatorial Mathematics, (Beckenbach, E., ed.) pp. 144-184. New York: Wiley. 